\title{
HIDROGÉIS HETEROGÊNEOS E HÍBRIDOS PARA APLICAÇÕES EM ENGENHARIA TECIDUAL
}

\author{
M. F. PASSOS ${ }^{1}$, C.G.B.T. DIAS ${ }^{2}$ e MACIEL FILHO, RUBENS ${ }^{3}$ \\ 1,3 Universidade Estadual de Campinas (UNICAMP), Faculdade de Engenharia Química, Laboratório \\ de Otimização, Projetos e Controle Avançado (LOPCA), Campinas, SP-Brasil \\ ${ }^{2}$ Universidade Federal do Pará (UFPA), Faculdade de Engenharia Mecânica, Belém, Pará-Brasil \\ E-mail para contato: cellepassos@hotmail.com
}

\begin{abstract}
RESUMO - A carência de enxertos disponíveis, na reparação de tecidos doentes e/ou danificados, assim como a ineficácia de alguns transplantes alogênicos, desperta o interesse dos pesquisadores para a geração de tecidos autólogos, através de novas abordagens em engenharia. Porém, esta não é uma tarefa fácil. O desenvolvimento destes materiais, visando Engenharia Tecidual (ET), leva em consideração a complexidade de órgãos e tecidos. A fim de solucionar este problema, hidrogéis poliméricos convencionais, homo ou copolímeros, vêm disputando espaço com hidrogéis heterogêneos, na busca incessante de obter biomateriais para aplicações médicas. Esta nova classe de hidrogéis confere propriedades específicas dos materiais combinados, permitindo melhora nas propriedades mecânicas e superficiais. A viabilidade dos mesmos engloba desde aplicação como scaffolds até sistema de liberação controlada de fármacos. A fim de elucidar as diferentes aplicações destes materiais em ET, este trabalho apresenta os fundamentos teóricos dos hidrogéis heterogêneos, derivados, principalmente, das redes inter e semiinterpenetrantes.
\end{abstract}

\section{INTRODUÇÃO}

A busca incessante de novos processos e tecnologias, a fim de obter produtos com características adequadas para aplicação em Engenharia Tecidual (ET), vêm integrando diversas áreas científicas. Biólogos, engenheiros, médicos, químicos e físicos, sintetizam, caracterizam e direcionam a aplicação dos biomateriais. Todo este esforço surge da carência de enxertos disponíveis na reparação de tecidos doentes ou danificados. Em alguns casos, transplantes alogênicos podem ser utilizados a fim de solucionar o problema. Mas, para situações que apresentam defeitos de tecidos altamente imunogênicos, como a pele, esta alternativa não é eficaz. Diante disso, torna-se necessário a geração de tecidos autólogos através de novas abordagens em engenharia (Liu e Cao, 2011). Porém, esta não é uma tarefa fácil. O desenvolvimento destes materiais visando medicina regenerativa leva em consideração a complexidade de órgãos e tecidos. Na produção de um coração ou fígado, são necessários múltiplos tipos de células e scaffolds adequados, devido às suas funções metabólicas complexas. Com o objetivo de minimizar ou solucionar tais problemas, a Engenharia Tecidual incentiva o desenvolvimento de diferentes biomateriais, bem como o processamento dos mesmos (Griffith , 2000). Os hidrogéis poliméricos tornam-se uma alternativa promissora e interessante. 


\section{9 a 22 de outubro de 2014 \\ Florianópolis/SC}

Hidrogéis são definidos como polímeros tridimensionais que possuem alta capacidade de absorção de água (>38\%) (Nogueira et al., 2012), sem se dissolver. Alguns dos atrativos destes materiais são: baixa irritação friccional; canais nano e micro-porosos para transporte de nutrientes; permeabilidade; consistência macia e elástica, semelhante aos tecidos vivos; facilidade de modificação da superfície com biomoléculas; e, biocompatibilidade (Remya e Nair, 2012; Patel e Mequanint, 2011). Comumente, são estudados no campo farmacêutico para liberação controlada de moléculas bioativas ou em engenharia tecidual para encapsulamento de células (Pescosolido et al., 2011).

A Tabela 1 sumariza alguns estudos com hidrogéis poliméricos, discorridos desde o trabalho pioneiro de Witcherle e Lim (1960).

Tabela 1 - Progresso dos Hidrogéis e suas Aplicações

\begin{tabular}{|c|c|}
\hline Hidrogéis & Aplicações \\
\hline Poli(2-hidróxietil metacrilato) (PHEMA) & Lentes de Contato (Witcherle e Lim, 1960) \\
Polinivil álcool (PVAl) & Cirurgias de reconstrução de articulações (Bray e \\
& Merryk, 1973) \\
Poli (acetato de vinila)-PVAc & Prótese (Sasada, 1988) \\
Polietilenoglicol e Polilactídeo & Liberação controlada de proteínas (Hiemstra et al., \\
& 2007) \\
Polivinil álcool e Polivinil pirrolidona & Reparo de cartilagem (Spiller et al., 2008) \\
Poli(2-hidróxietil metacrilato) (PHEMA) e & Engenharia Tecidual (Patel e Mequanint, 2011) \\
Poliuretano & Liberação controlada de pilocarpina ocular \\
Hidrogéis vinílicos contendo $\alpha-$ & (Casolaro et al., 2012) \\
aminoácidos & Encapsulamento de células (Chien et al., 2012) \\
Hidrogéis de carboxibetaína & Scaffolds (Chang et al., 2013) \\
Hidrogéis híbridos de quitina/ & \\
hidroxiapatita & \\
\hline
\end{tabular}

\section{HIDROGÉIS HETEROGÊNEOS/ HÍBRIDOS}

Após difusão, por Witcherle e Lim (1960), do hidrogel de poli (2-hidróxi etil metacrilato) PHEMA- para utilização como lentes de contato inúmeros esforços vêm sendo desenvolvidos na síntese de novos hidrogéis ou, ainda, aperfeiçoamento dos mesmos para fins biomédicos, como liberação controlada de fármacos ( Chang et al., 2013) e scaffolds ( Wu et al., 2011).

Hidrogéis heterogêneos, derivados de misturas poliméricas, copolímeros, redes interpenetrantes (IPN) ou semi interpenetrantes (semi-IPN), têm sido bastante investigados (Thankama et al., 2013; Kozhunova et al., 2012; Nizam El- Din e El-Naggar, 2012) Este fato pode ser elucidado perante a necessidade de satisfazer critérios como biodegradabilidade, propriedades mecânicas e interações polímeros-polímeros (funcionalidade adicional). Em alguns casos, estes materiais apresentem micro ou macro separação de fases, mas combinam propriedades físico-químicas de cada componente. 


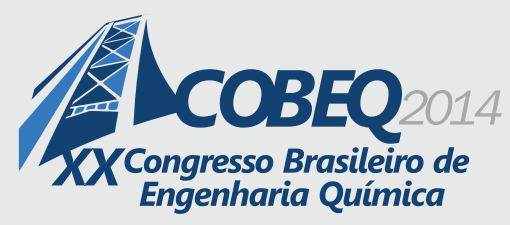

19 a 22 de outubro de 2014
Florianópolis/SC

Desta forma, é possível adaptar matrizes poliméricas para otimizar a liberação de drogas baseada em suas propriedades, dose e parâmetros farmacocinéticos (Bae e Kim, 1993).

\section{REDES SEMI-INTERPENETRANTES (SEMI-IPN/SIPN) E REDES INTERPENETRANTES (IPN): DEFINIÇÃO E CARACTERÍSTICAS}

Redes poliméricas interpenetrantes (IPN) ou semi-interpenetrantes (SIPN ou semi-IPN) recebem diferentes nomenclaturas na literatura e, em alguns casos, encontram-se divergências em suas definições. Alguns materiais descritos como semi-IPN estão covalentemente ligados ou recebem denominações de blendas poliméricas. Segundo a IUPAC, rede interpenetrante pode ser definida como: "um polímero compreendendo de duas ou mais redes que são pelo menos parcialmente entrelaçadas em uma escala molecular, mas não covalentemente ligados um ao outro e que não podem ser separados, a menos que as ligações químicas sejam quebradas". Uma mistura de duas ou mais redes poliméricas pré-formadas não é uma IPN (Loureiro et al., 2012; Wu et al., 2011). Esta última sentença salienta claramente a diferença entre IPN e blendas. O emaranhamento de dois polímeros com ligações cruzadas em uma IPN leva a uma "miscibilidade forçada", quando comparada com misturas de blendas poliméricas normalmente incompatíveis (Ratna e Karger-kocsis, 2011). À medida que os monômeros polimerizam, a miscibilidade dos componentes diminui, principalmente por razões entrópicas, podendo resultar na separação de fases. No entanto, este efeito é menos pronunciado com a interpenetração das redes, permitindo otimização e controle das propriedades físicas e mecânicas destes materiais. A transição vítrea tende a ser muito ampla, cobrindo o intervalo entre as temperaturas dos dois componentes poliméricos (Sangermano et al., 2012). Logo, a associação entre diferentes polímeros geralmente é resultante da necessidade em superar os pontos débeis dos materiais isolados, obtendo produtos com propriedades únicas, devido ao efeito sinérgico. (Wu et al, 2011; Chikh et al., 2011; Kozhunova et al, 2012).

Por outro lado, redes semi-interpenetrantes (semi-IPN), conhecidas desde 1960, é uma mistura polimérica, em princípio, que tem a possibilidade de separação de seus componentes sem que haja quebra de ligações químicas (Loureiro et al., 2012; Moszczyńsk et al., 2007). Diferem de IPN pela sua composição. São constituídas de um polímero linear entrelaçado na rede polimérica de outro polímero, cujo polímero linear pode, usualmente, ser extraído através da utilização de um solvente adequado. Segundo a IUPAC, redes semi-IPN são: "polímeros compreendendo de duas ou mais redes e um ou mais polímeros lineares ou ramificados. São caracterizados pela penetração em escala molecular de, pelo menos, uma das redes por, pelo menos, algumas das macromoléculas lineares ou ramificadas" (Chikh et al., 2011).

A associação com um polímero linear confere às redes semi-IPN inúmeras vantagens quando comparadas às redes interpenetrantes. A alta viscosidade das redes semi-IPN, a facilidade de manipulação e a possibilidade de cura ultravioleta tornam este tipo de sistema adequado à formação de hidrogéis in situ (Pescosolido et al., 2011). Conforme Chikh et al. (2011), em redes semi-IPN não há necessidade de funcionalidade do polímero linear. Isto permite maior flexibilidade na escolha do material de partida. Além disso, somente uma reação química é necessária na obtenção do material, reduzindo a ocorrência de sítios de reações indesejadas, como a copolimerização e/ou grafitização. No entanto, a arquitetura destas redes também possui desvantagens. Por definição, o polímero linear 


\section{9 a 22 de outubro de 2014 \\ Florianópolis/SC}

pode, potencialmente, ser extraído do material em condições que têm que ser determinadas e, em seguida, escrupulosamente evitadas. Acrescenta-se, ainda, certa possibilidade de separação de fases, a qual pode ser prejudicial para algumas aplicações.

\section{REDES IPN/ SEMI-IPN E HIDROGÉIS: APLICAÇÕES E SÍNTESE}

Várias pesquisas direcionadas a redes poliméricas semi-interpenetrantes (semi-IPN) e interpenetrantes (IPN) vêm sendo citadas na literatura (Delhorbe et al., 2013; Kozhunova et al., 2012; Sangermano et al., 2012; Pescosolido et al., 2011). Há diferentes formas de obtenção e aplicações finais. Materiais oriundos de géis/ hidrogéis são os principais polímeros sintetizados com redes IPN e semi-IPN a fim de se obter características viáveis em aplicações farmacêuticas e biomédicas. As propriedades resultantes dependem de inúmeros fatores, incluindo hidrofilicidade e grau de reticulação.

Hidrogéis IPN podem ser compostos por polivinil álcool (PVAl), quitosana, polietileno glicol (PEG), poli (2-hidróxietilmetacrilato) (PHEMA), etc. Pescosolido et al. (2011) estudaram hidrogéis interpenetrantes (IPN) in situ de alginato de cálcio e dextrano-HEMA. Segundo autores, estes materiais têm potencial para aplicação em medicina regenerativa, liberação de proteínas e aplicações em engenharia tecidual, devido às suas características mecânicas, de degradação, cinética e biocompatibilidade. É uma nova classe de hidrogéis baseados na intercalação de duas redes poliméricas diferentes. Nizam El-Din e El-Naggar (2012) sintetizaram estruturas poliméricas (hidrogéis) interpenetrantes de ácido acrílico/ polietilemoimina utilizando irradiação gama. Estudos indicaram viabilidade da liberação controlada de fármacos, dependente do pH. Silan et al. (2012) prepararam partículas de hidrogéis e filmes IPN dos mesmos. Estas espécies de materiais apresentaram potencial para aplicações como curativos e, as partículas sintetizadas, após modificação, apresentaram propriedades antimicrobianas contra algumas bactérias, tais como S. aureus ATCC6538, P. aeruginosa ATCC9027, B. subtilis ATCC6633, e E. coli ATCC8739.

Thankam et al. (2013) prepararam hidrogéis IPN biossintéticos de poli vinil álcool com alginato de cálcio. Os pesquisadores demonstraram a viabilidade do produto para engenharia de tecido cardíaco. Redes poliméricas IPN de nanogéis baseados em gelatina e poli (ácido acrílico) foram sintetizadas por Koul et al. (2011). Os resultados demonstraram uma estratégia aplicável para a síntese de nanogéis multifuncionais, mas restrito a interação adequada entre os componentes do sistema. Tais nanogéis apresentaram-se viáveis como carreadores de drogas no tratamento de tumores sólidos.

\subsection{Síntese das redes IPN/semi-IPN}

Redes IPN são usualmente sintetizadas pela polimerização de dois monômeros multifuncionais via mecanismos não-interferentes (catiônicos, aniônicos, radicalar etc.). Radiação visível ou ultravioleta (UV) também tem sido utilizada para produzir estes tipos de materiais. A polimerização fotoiniciada pode ser obtida através de uma mistura do monômero, acrilato e epóxido, onde o acrilato polimeriza por mecanismo radicalar, enquanto o epóxido polimeriza via mecanismo catiônico (Sangermano et al., 2012). 


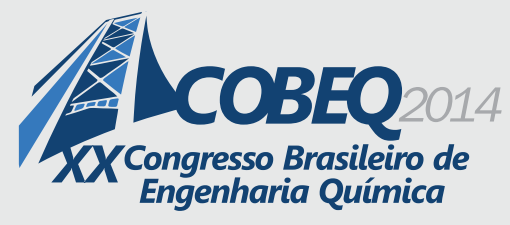

19 a 22 de outubro de 2014
Florianópolis/SC

Duas abordagens principais para a síntese de redes IPN são: (1) sequencial - é relatada quando a segunda rede é formada ao redor de uma rede primeiramente sintetizada; (2) simultânea - quando a polimerização e reticulação dos dois monômeros acontecem simultaneamente, mas por distintos mecanismos. Ambas as rotas são utilizadas a fim de obter materiais cujas redes poliméricas são entrelaçadas fisicamente a nível molecular (Kozhunova et al., 2012).

A síntese de redes semi-interpenetrantes é ligeiramente diferente do procedimento IPN. Neste caso, um dos componentes não sofre reticulação, podendo estar polimerizado ao adicionar a solução do segundo componente. Em algumas situações, as cadeias lineares introduzidos na rede reticulada podem ser facilmente funcionalizadas (Kozhunova et al., 2012). Reações de polimerização/ reticulação levam a síntese de polímeros lineares ou reticulados, geralmente obtidos por radicais livres, iniciados por fótons ou processos térmicos (Chikh et al., 2011).

Caminhos para a obtenção das redes IPN e semi-IPN são relatados por Chikh et al. (2011) e estão demonstradas na Figura 1. No processo simultâneo, todos os reagentes são misturados antes de ativar qualquer mecanismo de polimerização ou reticulação. Neste caso, a morfologia do material obtido pode ser modulada e altamente diferente conforme proporções dos dois polímeros, ordem ou taxa relativa da formação das duas redes. Na síntese sequencial, a primeira rede polimérica é sintetizada e,subsequentemente, inchada em todos os precursores necessários para a formação da segunda rede, que por sua vez, determina a morfologia do material final.

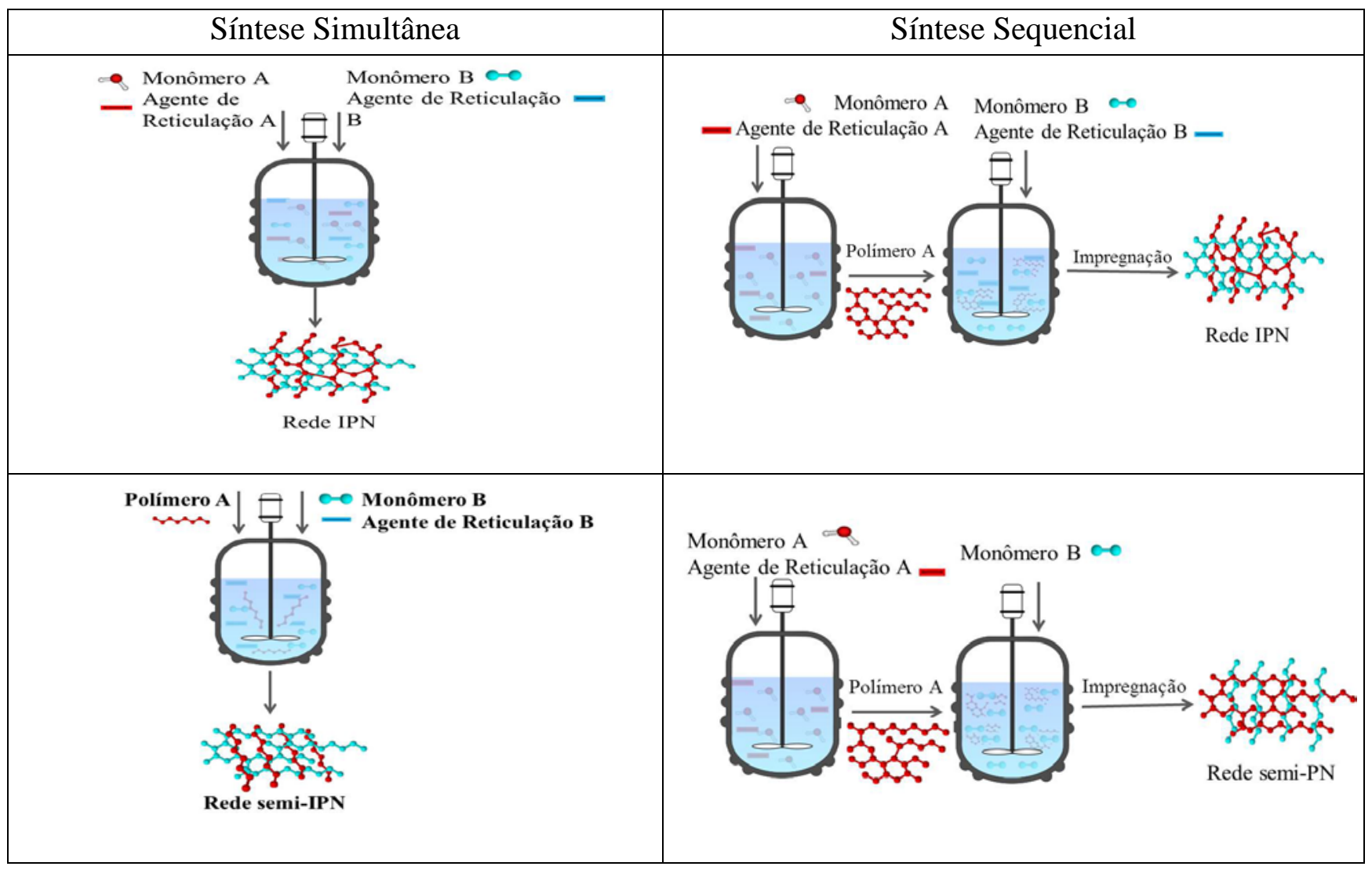

Figura 1- Caminhos para a obtenção das redes IPN e semi-IPN. 


\section{9 a 22 de outubro de 2014 \\ Florianópolis/SC}

\section{HIDROGÉIS COMPÓSITOS E/OU HÍBRIDOS}

A combinação entre materiais inorgânicos e polímeros tem sido cada vez mais utilizada na obtenção de compósitos ou híbridos. Os híbridos compreendem redes inorgânicas homogêneas interdispersas ou interpenetradas em uma matriz polimérica (Vallés-lluch et al., 2010a). Os hidrogéis compósitos consiste de um polímero com partículas ou fibras, fornecendo uma estrutura capaz de intumescer em água ( Bae e Kim, 1993). Um exemplo de híbrido é a combinação do hidrogel de poli 2-hidróxietil metacrilato (PHEMA) com sílica. O PHEMA, além de ser um biomaterial biocompatível, de fácil manipulação e bastante explorado no campo científico, fornece grupos hidroxilas que podem formar ligações de hidrogênio e ligações covalentes do tipo Si-O-C, pela condensação com grupos silanóis (Si-OH) da sílica (Costa e Vasconcelos, 2002). Por outro lado, a sílica atua como reforço à matriz orgânica, melhorando suas propriedades mecânicas e conferindo bioatividade. Isso poderá fornecer aos materiais melhor interação com as células (Vallés-lluch et al., 2010a).

Compósitos PHEMA/sílica podem ser produzidos ou pela adição de nanopartículas de sílica ao monômero/PHEMA em solução ou usando soluções de PHEMA e precursores inorgânicos como o tetraetoxisilano (TEOS) através do processo sol-gel in situ. Estes materiais combinam as propriedades de inchamento do PHEMA e a bioatividade da $\mathrm{SiO}_{2}$, podendo construir um grupo de materiais artificiais para aplicações como scaffolds em ET ou para substituição de tecidos macios (Costantini et al., 2008).

Em ambientes fisiológicos (pH 7,4), a sílica é carregada negativamente e grupos silanóis são formados na superfície, por troca de íons alcalinos com prótons do meio. A carga negativa dos grupos silanóis da superfície interage com íons de cálcio positivo, formando compostos de cálcio, tais como silicato de cálcio amorfo. Estes compostos de cálcio possuem carga positiva e, conseqüentemente, combinam com os íons fosfatos negativos do fluido, formando um fosfato de cálcio amorfo, o qual cristaliza formando hidroxiapatita similar ao osso (Vallés-lluch et al, 2010b) Isto enfatiza os estudos polímero/sílica para aplicações médicas (Costa e Vasconcelos, 2002; Costantini et al., 2008; Fukushima et al., 2011; Vallés-lluch et al., 2009). A incorporação de uma fase inorgânica em uma matriz polimérica orgânica tende a imitar a composição dos tecidos mineralizados, onde a fase hidroxiapatita inorgânica está dispersa em uma matriz de colágeno, com o qual interage em um nível molecular produzindo um composto natural. Scaffolds porosos de compósitos híbridos imitando estruturas ósseas naturais têm sido preparados e investigados para servir de suporte ao crescimento e regeneração de tecidos (Vallés-lluch et al., 2010a).

Uma série de compósitos de poli(butileno succinato)(PBS)/sílica foram preparados por polimerização in situ, demonstrando dependência entre composição e biodegradabilidade, a fim de reduzir o ataque microbiano (Han et al., 2008). Vallés-Lluch et al., (2010a) sintetizaram compósitos de $\mathrm{P}(\mathrm{EMA}-\mathrm{co}-\mathrm{HEA}) / \mathrm{SiO}_{2}$ através do processo sol-gel. Segundo autores, os materiais obtidos apresentaram potencial para cultura de células, mantendo o fenótipo das células de polpa dentária, in vitro. Zhang et al. (2013) demonstraram um método para obter compósitos em larga quantidade e a custo baixo. Baseado neste procedimento, os polímeros resultantes obtiveram resposta a estímulos e estrutura assimétrica, apresentando viabilidade para aplicação como carreadores de drogas. 


\section{9 a 22 de outubro de 2014 \\ Florianópolis/SC}

\section{CONCLUSÃO}

Hidrogéis poliméricos embora conhecidos por vários anos, ainda tem atraído grande interesse no campo científico. A combinação dessas matrizes poliméricas, com partículas ou fibras e diferentes polímeros, associados, ainda, a novas tecnologias, possibilitam uma melhora considerável nas propriedades dos materiais sintetizados. Atributos mecânicos, bioatividade, degradabilidade e meios compatíveis para encapsulamento de moléculas biológicas ou medicamentos, são algumas vantagens destes biomateriais. É possível obter scaffolds com propriedades superficiais adeptas ao ataque e proliferação celular, além de polímeros com propriedades similares à estrutura óssea. Trata-se de um grupo promissor de materiais que inspira pesquisadores para futuras aplicações na área biomédica. Contudo, novas abordagens para a obtenção de hidrogéis heterogêneos se tornam imprescindíveis, no intuito de satisfazer a complexidade do corpo humano.

\section{REFERÊNCIAS}

BAE, Y. H.; KIM, S. W. Hydrogel delivery systems based on polymer blends, block co-polymers or interpenetrating networks. Advanced Drug Delivery Reviews 11: 109-135, 1993.

BRAY, J.C.; MERRYL, E.W. Poly (vinyl alcohol) hydrogels for synthetic articular cartilage material. Journal of biomedical Materials Research, 7, p.431-443, 1973.

CASOLARO, M.; CASOLARO, I.; LAMPONI, S. Stimuli-responsive hydrogels for controlled pilocarpine ocular delivery. - European Journal of Pharmaceutics and Biopharmaceutics 80: 553-561, 2012.

CHANG, C.; PENG, N.; HE, M.; TERAMOTO, Y.; NISHIO, Y.; ZHANG, L. Fabrication and properties of chitin/hydroxyapatite hybrid hydrogels as scaffold nano-materials. - Carbohydrate Polymers 91: 7-13, 2013.

CHIEN, H.-W.; TSAI, W.-B.; JIANG, S. Direct cell encapsulation in biodegradable and functionalizable carboxybetaine hydrogels. Biomaterials 33: 5706-5712, 2012.

CHIKH, L.; DELHORBE, V.; FICHET, O. (Semi-)Interpenetrating polymer networks as fuel cell membranes. - Journal of Membrane Science 368: 1-17, 2011.

COSTA, R. O. R.; VASCONCELOS, W. L. Structural modification of poly(2-hydroxyethyl methacrylate)-silica hybrids utilizing 3methacryloxypropyltrimethoxysilane. Journal of Non-Crystalline Solids, 304, p. 84-91, 2002.

COSTANTINI, A.; LUCIANI, G.; SILVESTRI, B.; TESCIONE, F.; BRANDA, F. Bioactive poly(2-hydroxyethylmethacrylate)/silica gel hybrid nanocomposites prepared by sol-gel process. Journal of Biomedical Materials Research Part B: Applied Biomaterials , vol. 86B issue 1, p. 98-104, 2008.

DELHORBE, V.; CAILLETEAUB, C.; CHIKH, LINDA.; GUILLERMO, A.; GEBEL, G.; MORIN, A.; FICHET, O. Influence of the membrane treatment on structure and properties of sulfonated poly(etheretherketone)semi-interpenetratingpolymernetwork. Journal of Membrane Science, 427, p. 283-292, 2013.

FUKUSHIMA, K.; TABUANI, D.; ABBATE, C.; ARENA, M.; RIZZARELLI, P. Preparation, characterization and biodegradation of biopolymer nanocomposites based on fumed silica. - European Polymer Journal 47: 139-152, 2011.

THANKAM G., F.; MUTHU, J.; SANKAR, V.; GOPAL, K. R. Growth and survival of cells in biosynthetic poly vinyl alcohol-alginate IPN hydrogels for cardiac applications. - Colloids and Surfaces B: Biointerfaces 107: 137-145, 2013.

GRIFFITH, L. G. Polymeric biomaterials. - Acta Materialia 48: 263-277, 2000.

HAN, S.-I.; LIM, J. S.; KIM, D. K.; KIM, M. N.; IM, S. S. In situ polymerized poly(butylene succinate)/silica nanocomposites: Physical properties and biodegradation. - Polymer Degradation and Stability 93: 889-895, 2008.

HIEMSTRA, C.; ZHONG, Z.; VAN TOMME, S. R.; VAN STEENBERGEN, M.J; JACOBS, J.J,; OTTER, W.D., HENNINK,W.E.;FEIJEN, J. In vitro and in vivo protein delivery from in situ forming poly(ethylene glycol)-poly(lactide) hydrogels. Journal of Controlled Release 119: 320-327, 2007. 


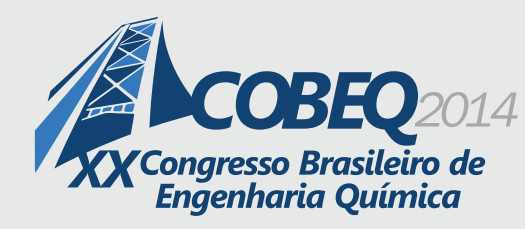

KOUL, V.; MOHAMED, R.; KUCKLING, D.; ADLER, H.-J. P.; CHOUDHARY, V. Interpenetrating polymer network (IPN) nanogels based on gelatin and poly(acrylic acid) by inverse miniemulsion technique: Synthesis and characterization. - Colloids and Surfaces B: Biointerfaces 83: 204-213, 2011.

KOZHUNOVA, E. Y.; MAKHAEVA, E. E.; KHOKHLOV, A. R. Collapse of thermosensitive polyelectrolyte semi-interpenetrating networks. - Polymer 53: 2379-2384, 2012.

LIU, W.; CAO, Y. 5.28 - Tissue-Engineering Technology for Tissue Repair and Regeneration. In: Editor-in-Chief: Murray, M.-Y., (Ed.) Comprehensive Biotechnology (Second Edition), pp. 353-375. Academic Press, Burlington, 2011.

LOUREIRO, F.A.M.; PEREIRA, R.P.; ROCCO, A.M. - “Cinética de cura de redes poliméricas semi-interpenetrantes baseadas em bisfenol-a e PEI”, in: XIX Congresso Brasileiro de Engenharia Química (COBEQ), Búzios-RJ, p. 7763- 7771, 2012.

MOSZCZYŃSKI, P.; KALITA, M.; PARZUCHOWSKI, P.; SIEKIERSKI, M.; WIECZOREK, W. Interpenetrating ionomer-polymer networks obtained by the in situ polymerization in pores of PVdF sponges as potential membranes in PEMFC applications, Journal Power Sources, 173, p. 648, 2007.

NIZAM EL-DIN, H. M. M.; EL-NAGGAR, A. W. M. Radiation synthesis of acrylic acid/polyethyleneimine interpenetrating polymer networks (IPNs) hydrogels and its application as a carrier of atorvastatin drug for controlling cholesterol. - European Polymer Journal 48: 1632-1640, 2012.

NOGUEIRA, N.; CONDE, O.; MIÑONES, M.; TRILLO, J. M.; MIÑONES JR, J. Characterization of poly(2-hydroxyethyl methacrylate) (PHEMA) contact lens using the Langmuir monolayer technique. - Journal of Colloid and Interface Science 385: 202210, 2012.

PATEL, A.; MEQUANINT. K. Synthesis and characterization of polyurethane-block-poly (2-hydroxyethyl methacrylate) hydrogels and their surface modification to promote cell affinity. Journal of Bioactive and Compatible Polymers, 26, p. 114- 129, 2011.

PESCOSOLIDO, L.; VERMONDEN, T.; MALDA, J.; CENSI, R.; DHERT, W.J.; ALHAIQUE, F.; HENNINK, W.E.; MATRICARDI, P. In situ forming IPN hydrogels of calcium alginate and dextran-HEMA for biomedical applications. - Acta Biomaterialia 7: 16271633, 2011.

RATNA, D.; KARGER-KOCSIS, J. Shape memory polymer system of semi-interpenetrating network structure composed of crosslinked poly (methyl methacrylate) and poly (ethylene oxide). - Polymer 52: 1063-1070, 2011.

REMYA, N. S.; NAIR, P. D. Engineering cartilage tissue interfaces using a natural glycosaminoglycan hydrogel matrix- an invitro study. - Materials Science and Engineering: C., 2012.

SANGERMANO, M.;COOK, W. D.; PAPAGNA, S.; GRASSINI, S. Hybrid UV-cured organic-inorganic IPNs. - European Polymer Journal 48: 1796-1804, 2012.

SASADA, T. - Abstracts of Third World Biomaterials Congress. Biomechanics and Biomaterials, 6, p. 21-25, 1988.

SILAN, C.; AKCALI, A.; OTKUN, M. T.;OZBEY, N.; BUTUN, S.; OZAY, O.; SAHINER, N. Novel hydrogel particles and their IPN films as drug delivery systems with antibacterial properties. - Colloids and Surfaces B: Biointerfaces 89: 248-253, 2012.

SPILLER, K. L.; LAURENCIN, S. J.; CHARLTON, D.; MAHER, S. A.; LOWMAN, A. M. Superporous hydrogels for cartilage repair: Evaluation of the morphological and mechanical properties. - Acta Biomaterialia 4: 17-25, 2008.

VALLÉS-LLUCH, A.; COSTA, E.; GALLEGO FERRER, G.; MONLEÓN PRADAS, M.; SALMERÓN-SÁNCHEZ, M. Structure and biological response of polymer/silica nanocomposites prepared by sol-gel technique. - Composites Science and Technology 70: 1789-1795, 2010a.

VALLÉS-LLUCH, A.; FERRER, G. G.; PRADAS M. M. Surface modification of P(EMA-co-HEA)/SiO2 nanohybrids for faster hydroxyapatite deposition in simulated body fluid? .Colloids and Surfaces B: Biointerfaces 70(2): 218-25, 2009.

VALLÉS-LLUCH, A.; RODRÍGUEZ-HERNÁNDEZ, J. C.; FERRER, G. G.; PRADAS, M. M. Synthesis and characterization of poly(EMA-co-HEA)/SiO2 nanohybrids. European Polymer Journal, 46, p. 1446-1455, 2010 b.

WITCHERLE, O.; LIM, D. Hydrophilic gels for biological use. Nature, v.185, p. 117-118,1960.

WU, W.; LIU, J.; CAO, S.; TAN, H.; LI, J.; XU, F.; ZHANG, X. Drug release behaviors of a pH sensitive semi-interpenetrating polymer network hydrogel composed of poly(vinyl alcohol) and star poly[2-(dimethylamino)ethyl methacrylate]. - International Journal of Pharmaceutics 416: 104-109, 2011.

ZHANG, X.;GAO, C.; LIU, M.;HUANG, Y.; YU, X.; DING, E. Synthesis and characterization of asymmetric polymer/inorganic nanocomposites with pH/temperature sensitivity. - Applied Surface Science, 264, p. 636-643, 2013. 